\title{
The Randomized Oslo Study of Renal Denervation vs. Antihypertensive Drug Adjustments: Efficacy and Safety Through 7 Years of Follow-up
}

Ola Undrum Bergland ${ }^{1,2}$, Camilla Lund Søraas ${ }^{1,3}$, Anne Cecilie K. Larstorp ${ }^{1,2,4}$, Lene V. Halvorsen ${ }^{1,2,5}$, Ulla Hjørnholm ${ }^{1}$, Pavel Hoffman ${ }^{6}$, Aud Høieggen ${ }^{1,2,5}$, Fadl Elmula M. Fadl Elmula 1,7

${ }^{1}$ Section for Cardiovascular and Renal Research, Oslo University Hospital Ullevål, Oslo, Norway

${ }^{2}$ Institute of Clinical Medicine, University of Oslo, Oslo, Norway

${ }^{3}$ Section for Environmental and Occupational Medicine, Oslo University Hospital Ullevål, Oslo, Norway

${ }^{4}$ Department of Medical Biochemistry, Oslo University Hospital Ullevål, Oslo, Norway

${ }^{5}$ Department of Nephrology, Oslo University Hospital Ullevål, Oslo, Norway

${ }^{6}$ Section for Interventional Cardiology, Department of Cardiology, Oslo University Hospital Ullevål, Oslo, Norway

${ }^{7}$ Department of Acute Medicine, Oslo University Hospital Ullevål, Oslo, Norway

\section{Corresponding Author}

Dr. Ola Undrum Bergland

Section for Cardiovascular and Renal Research, Oslo University Hospital Ullevål, Oslo, Norway, Box 4950 Nydalen, 0424 Oslo, Norway

oluber@ous-hf.no

$+4722119338$

Clinical Trial Number registered at www.clinicaltrials.gov: NCT01673516

Word Count: 292 Abstract; 3.036 for main text +35 references +3 tables +3 figures 


\section{ABSTRACT}

Purpose: The blood pressure (BP) lowering effect of renal sympathetic denervation (RDN) in treatment-resistant hypertension shows variation amongst the existing randomized studies. The long-term efficacy and safety of RDN requires further investigation. For the first time, we report BP changes and safety up to seven years after RDN, compared to drug adjustment in the randomized Oslo RDN study.

Materials and methods: Patients with treatment-resistant hypertension, defined as daytime systolic ambulatory BP $\geq 135 \mathrm{mmHg}$ after witnessed intake of $\geq 3$ antihypertensive drugs including a diuretic, were randomized to either RDN $(n=9)$ or drug adjustment $(n=10)$. The initial primary endpoint was the change in office BP after six months. The RDN group had their drugs adjusted after one year using the same principles as the Drug Adjustment group. Both groups returned for long-term follow-up after three- and seven years.

Results: The decrease in office BP and ambulatory BP (ABPM) after six months did not persist, but gradually increased in both groups. From six months to seven years follow-up, mean daytime systolic ABPM increased from $142 \pm 10$ to $145 \pm 15 \mathrm{mmHg}$ in the RDN group, and from $133 \pm 11$ to $137 \pm 13 \mathrm{mmHg}$ in the Drug Adjustment group, with the difference between them decreasing. In a mixed factor model, a significantly different variance was found between the groups in daytime systolic ABPM ( $p=0.04)$ and diastolic ABPM ( $p=0.01)$ as well as office diastolic BP $(p<0.01)$, but not in office systolic BP $(p=0.18)$. At long-term followup we unveiled no anatomical- or functional renal impairment in either group.

Conclusion: Blood pressure changes up to seven years show a tendency towards a smaller difference in BPs between the RDN and drug adjustment patients. Our data support RDN as a safe procedure, but it remains non-superior to intensive drug adjustment seven years after the intervention.

\section{KEYWORDS}

Treatment-resistant hypertension. Renal Denervation. Long-term follow-up. Safety. 


\section{INTRODUCTION}

Over the last decade, renal sympathetic denervation (RDN) has emerged as a treatment option for arterial hypertension, even showing promising results in patients who are seemingly treatment-resistant (1). A few published trials showed promising results of RDN with modest BP-lowering effect in selected individuals (2). After the initial proof-of-concept studies Symplicity HTN-1 and Symplicity HTN-2 sparked great interest in invasive treatment of hypertension, the sham-controlled Symplicity HTN-3 caused some controversy after it failed to demonstrate RDN superiority in reducing daytime ABPM six months after the procedure $(3,4)$. The more recent proof-of-concept-trials SPYRAL HTN-ON-MED and SPYRAL HTN OFF-MED showed promising results, however with modest effect comparable to that achieved by a single antihypertensive drug (5-7). As with all novel treatment methods, efficacy and safety of RDN need close follow-up in regard to any potential complications of both short- and long-term. A previously published meta-analysis revealed RDN as a safe procedure up to six months, while more recently published data from a large cohort suggests that the reduction in office- and ambulatory BP is sustained up to three years without any deterioration in renal function (8-10). Follow-up data on outcomes and safety beyond three years are lacking (11). In the present study, we aimed to describe long-term follow-up data of the participants in the randomized Oslo RDN study after three- and seven years to assess both efficacy and safety of renal denervation, providing additional knowledge of RDN compared to optimized drug treatment.

\section{MATERIAL AND METHODS}

\section{Procedures and treatments}


The design and rationale of the Oslo RDN study (Fig. 1) have been described in detail (12, 13). In short, of 65 patients with apparent treatment-resistant hypertension who were referred for RDN, 45 patients were excluded after a thorough investigation, either due to secondary causes or after obtaining a normalized daytime ABPM following witnessed intake of drugs as previously described by us and detailed in the Online Supplement (14). Thus, 20 patients were randomized to either RDN or drug adjustment assisted by hemodynamic assessments using the HOTMAN system (Hemo Sapiens Inc. Sedona, AZ, USA), described in detail by others (15). Treatment was at all times in accordance with the latest guidelines of the European Society of Hypertension/European Society of Cardiology Guidelines on arterial hypertension (16-18). One patient from the RDN group was diagnosed with Conn's syndrome after randomization, and was subsequently excluded. All patients were planned for long-term follow-up after three- and seven years.

Both office- and ABPM were measured in a standardized manner according to existing guidelines, using Microlife WatchBP O3 (Microlife Health Management Ltd, Cambridge, UK). Witnessed intake of drugs was performed at follow-up visits, immediately prior to all 24 hour ABPM measurements. Ambulatory measurements were performed every 20 min during daytime, and every 30 min during the night. Blood- and urine samples were collected from patients at all follow-up visits. The estimated glomerular filtration rate (eGFR) was used to detect any decline in renal function, and was calculated using the CKD-EPI (Chronic Kidney Disease Epidemiology Collaboration) formula for creatinine $(19,20)$. In patients with extremes in body size or muscle mass we used Cystatin C to estimate kidney function, as the eGFR based on creatinine would give an inaccurate estimation (21). 
All patients underwent either computed tomography or magnetic resonance imaging close to randomization, and after three- and seven years. These imaging studies were done at the Dpt. of Radiology at our hospital. Patients were instructed to report adverse events related to RDN or drug adjustment.

\section{Ethical approval}

The regional ethical committee for medical and health research approved the Oslo RDN study. All patients gave their written informed consent for participation. The trial is registered with ClinicalTrials.gov with the NCT Number NCT01673516. Data may be made available upon request.

\section{Statistical analyses}

Statistical analyses were performed using IBM SPSS Statistics 25 (SPSS, Chicago, IL, USA), while analyses of variance were performed with JMP 14.0 (SAS, Cary, NC, USA) under guidance of an experienced statistician. Variables were tested for normality using the Kolmogorov-Smirnov test. For comparison of normally distributed data between the groups, Independent Samples t-test was used, along with Mann-Whitney U-test for the nonparametric data. For longitudinal data within both groups, we used Paired Samples t-test and Wilcoxon Signed Rank Test accordingly. Nominal data were assessed using the Fisher's Exact Probability test. A one-way repeated measure ANOVA (or the Friedman test as a nonparametric alternative) was used to investigate the effect of time on BP within each group, along with a mixed model analysis of variance applied for simultaneous testing of all followups for both office- and ABPM, respectively. A 2-sided $\alpha$-level of 0.05 was used in all 
superiority testing. All continuous variables are presented as Mean (SD), while categorical values are presented as percentages unless otherwise specified.

\section{RESULTS}

\section{Patient characteristics}

Background characteristics and comorbidity of the patients are summarized in Table 1. The RDN group included nine patients; seven males and two females, while the Drug Adjustment group had only male patients. Patients in the Drug Adjustment group had a slightly higher, although not significantly, mean age at inclusion (62.7 vs. 57.0, p=0.16), and this group carried more comorbidity at inclusion.

\section{Medication}

All patients included in the Oslo RDN study were treated in accordance with the 2007

ESH/ESC guidelines, and later the 2013- and 2018 guidelines $(17,18)$. At randomization, they used maximally tolerated doses of $\geq 3$ antihypertensive drugs including at least one diuretic. Some patients, however, used more than one agent from the same drug class. A detailed overview of antihypertensive treatment is shown in Table 2.

\section{One-year follow-up}

All patients in the RDN group who still had uncontrolled hypertension after one year $(n=7)$, underwent the same hemodynamic assessment as the Drug Adjustment group, and had their medications changed accordingly. In total, three patients did not attend the one-year followup, while another three had this follow-up substantially delayed, making these data 
incomplete and less consistent. As such, the one-year follow-up data were not included in the statistical analyses of variance.

Regardless, the office BP $( \pm S D)$ in the RDN group $(149 \pm 11 / 91 \pm 12 \mathrm{mmHg})$ and the Drug Adjustment group $(139 \pm 15 / 83 \pm 11 \mathrm{mmHg}$ ) were not significantly different $(p=0.15 / 0.48)$. However, the daytime ABPM in the RDN group $(145 \pm 14$ / $87 \pm 4 \mathrm{mmHg})$ significantly differed from that of the Drug Adjustment group $(134 \pm 12 / 78 \pm 6 \mathrm{mmHg})$, both in respect to systolic BP $(p=0.04)$ and diastolic BP $(p=0.004)$. Mean eGFR in the RDN group was $90.1 \pm 10.6$ vs. $85.2 \pm 9.2 \mathrm{~mL} / \mathrm{min} / 1.73 \mathrm{~m}^{2}$ in the Drug Adjustment group, $\mathrm{p}=0.29$. This is consistent with the trend observed from both the six month- and three-year follow-up.

\section{Long-term changes in office blood pressure}

Office BP (Fig. 2) decreased significantly in both groups after six months. This reduction was more prominent in the Drug Adjustment group (-28/-11 mmHg) compared to the RDN group $(-8 /-2 \mathrm{mmHg})$. However, the office BP in both groups increased over time. Within the RDN group, a one-way repeated measures ANOVA revealed no significant effect of time on either mean systolic- or diastolic BP (156/91 $\mathrm{mmHg}$ at randomization, $148 / 89 \mathrm{mmHg}$ at six months, $147 / 88 \mathrm{mmHg}$ at three years and $155 / 93 \mathrm{mmHg}$ at seven years, $\mathrm{p}=0.44 / 0.68)$. Similarly, this trend was also observed in the Drug Adjustment group, although the effect on mean diastolic BP was borderline significant $(160 / 88 \mathrm{mmHg}$ at randomization, $132 / 77 \mathrm{mmHg}$ at six months, $148 / 86 \mathrm{mmHg}$ at three years, and 146/85 $\mathrm{mmHg}$ at seven years, $\mathrm{p}=0.09 / 0.06$ ). Applying a mixed factor model where all follow-up visits are tested simultaneously, the two groups did not statistically differ in systolic BP $(p=0.18)$, but differed in diastolic BP $(p \leq 0.01)$. 


\section{Long-term changes in ambulatory blood pressure}

ABPM (Fig. 3) showed a similar trend between the groups. The RDN group had a further reduction of both daytime systolic- and diastolic ABPM from randomization up to the threeyear follow-up, before it increased at the seven-year follow-up. Within the RDN group, a one-way ANOVA revealed no significant effect of time in either systolic BP or diastolic BP (152/93 $\mathrm{mmHg}$ at randomization, $142 / 86 \mathrm{mmHg}$ at six months, $139 / 83 \mathrm{mmHg}$ at three years, and $145 / 87 \mathrm{mmHg}$ at seven years, $\mathrm{p}=0.18$ / 0.16). The decrease in the Drug Adjustment group was more pronounced after six months, while both systolic- and diastolic BP was modestly increased at the long-term follow-up. A one-way ANOVA showed a significant difference in effect of time on both systolic BP and diastolic BP $(152 / 88 \mathrm{mmHg}$ at randomization, $133 / 77 \mathrm{mmHg}$ at six months, $134 / 80 \mathrm{mmHg}$ at three years, and 137/82 $\mathrm{mmHg}$ at seven years, $\mathrm{p}=0.02$ / 0.01). For in-between groups comparison, the mixed factor model showed that both daytime systolic ABPM $(p=0.04)$ and diastolic ABPM $(p=0.01)$ statistically differed.

24-hour diastolic ABPM ( $p<0.01)$ and nighttime diastolic ABPM $(p<0.01)$ were statistically different between the two groups after six months, otherwise no differences were found on long-term follow-up.

\section{Biochemical assessments}

The two groups were highly comparable both before randomization (differing only in LDL cholesterol) and at all follow-up visits (differing only in C-Reactive Protein after seven years) as seen in Table 3. More details can be found in the Online Supplement. 


\section{Radiological examinations}

Computed tomography or magnetic resonance imaging revealed no cases of renal artery stenosis or other acquired changes after seven years.

\section{Adverse events in the RDN group}

We found no complications related to the RDN procedure. Four patients experienced adverse events of other causes that were treated accordingly. One patient was diagnosed with a Conn adenoma approximately two years after randomization and underwent unilateral adrenalectomy further complicated by a pulmonary embolism. The second patient experienced a substantial office BP reduction (177/108 mmHg at randomization vs. 142/91 $\mathrm{mmHg}$ after six months) with subsequent dizziness/lightheadedness and orthostatic hypotension. This resolved after reduction of antihypertensive medication. Further, the same patient experienced chest pain that was diagnosed as coronary artery spasms. The third patient was diagnosed with localized malignant melanoma. The fourth patient had a myocardial infarction.

\section{Adverse events in the Drug Adjustment group}

Seven patients in the Drug Adjustment group experienced adverse events and received appropriate treatment. These events were unrelated to study participation. The first patient experienced a fall accident with only minor head trauma approximately two years after

randomization, causing sequelae of chronic tiredness. The same patient died from complications of emphysema seven years after randomization. The second patient was 
diagnosed with claudication. The third- and fourth patients were diagnosed with benign prostate hyperplasia, while one of them was additionally found to have sleep apnea. The fifth patient was diagnosed with primary hyperaldosteronism (without Conn adenoma) approximately one year after randomization, and was treated with an aldosterone antagonist. The last two patients were diagnosed with atrial fibrillation.

\section{DISCUSSION}

As illustrated in the original publication, the BP-lowering effect of RDN was not superior to drug adjustment after six months (12). Analysis of variance (ANOVA) during seven year follow-up showed a significantly lower trend for systolic- and diastolic $24 \mathrm{~h}$ ABPM, daytime ABPM as well as office diastolic BP in the Drug Adjustment group. However, there was no difference between groups in office systolic BP over time. Despite the limited sample size in the study, the statistical method applied is reliable (22). Long-term development of office BP (Fig. 2) and ABPM (Fig. 3) showed that the two groups became more similar over time. It is interesting; however, that the RDN group maintained a slow decrease in both office- and ABPM between the six months- and three-year follow-up while the Drug Adjustment group experienced a slight increase in the same time period. The Drug Adjustment group better maintained their office- and ambulatory BP than the RDN group. A possible explanation for this could be that although the RDN group had their drugs adjusted after one year, they only returned for their next follow-up appointment six months after. As such, they did not receive the same intensity of follow-up after this adjustment as the Drug Adjustment group did in the first six months of the study. Given more attention in the last few years, such intensive follow-up, along with reduction of time to reach BP goal, may improve treatment of hypertension $(23,24)$. Whether this represents a longer-lasting effect of RDN compared to 
drug adjustment, or the two groups regress toward the mean at different paces remains a subject of discussion.

It is important to consider any decline in renal function when evaluating the long-term safety of RDN. Since eGFR is a more robust measure of renal function than serum creatinine alone, we used this as a measure of renal function (25). Among our patients, we found one statistical outlier in the RDN group at randomization. It is well known that the CKD-EPI formula for calculating the eGFR from creatinine is not ideal for all ages and body compositions, which could explain the finding in this particular patient (26). After careful consideration, this patient was not included in the statistical analysis of eGFR to achieve a more appropriate comparison of the groups.

Any long-term effect in renal function following RDN may be obscured by both the drug adjustment after one year, as well as changes in medication made by the patients' primary physician in between the visits. In any regard, it does not appear that the eGFR of either group decreased at a more rapid rate than can be expected from an aging population of patients with treatment-resistant hypertension (27-29). It is worth noting that the eGFR in the RDN group has declined slower than that of the Drug Adjustment group. Contributing factors to this may be lower mean age, a reduction in number of drugs, or even the longterm reduction of hypertensive stress on the kidneys (30). This also opens up the possibility of RDN being indirectly renoprotective by reducing the overall use of drugs that may interfere with normal renal function.

The two groups were similar in their characteristics at randomization. The mean age in the Drug Adjustment group was slightly higher, although not significantly, than in the RDN 
group, and they carried more comorbidity. This made the Drug Adjustment group more likely to be treated with cholesterol-lowering drugs, providing a possible explanation for the difference in LDL cholesterol at randomization. This difference vanished in time, leaving the two groups statistically indistinguishable from each other in regards to biochemical assessment on long-term follow-up.

All patients participating in the Oslo RDN Study had apparent treatment-resistant hypertension. Despite using maximally tolerated doses of $\geq 3$ antihypertensive drugs - one of which had to be a diuretic - they all remained hypertensive on daytime ABPM following witnessed intake of already prescribed drugs. Even though witnessed intake was used to verify medication adherence prior to ABPM at all follow-up visits, this did not eliminate the potential effects of non-adherence. As it might take several half-lives of a given drug in order to reach a pharmacokinetic steady-state, and thus full effect, a single intake is reliable only for the day it is performed (31). Even though indirect methods for detecting non-adherence are helpful, they are less accurate than direct methods - in particular biochemical quantification of drugs in serum or urine $(32,33)$. Interestingly, the total number of antihypertensive agents decreased in both groups after seven years. This reduction was larger in the RDN group (6.0 agents at randomization and 3.9 agents after seven years) than in the Drug Adjustment group (5.0 at randomization vs. 4.7 agents after seven years). The larger reduction in the RDN group was mainly driven by two patients; of which the first of which may have had a pronounced response to RDN, using 10 antihypertensive drugs at randomization, two drugs after three years, and only one drug at seven-year follow-up. The second patient used five antihypertensive drugs at randomization and after three years, but had since discontinued all drugs because of intolerable side effects. 
Medication optimization guided by HOTMAN differs from that of real life conditions. Although very few studies on this subject exist, a randomized controlled study on 167 patients revealed no difference between HOTMAN-assisted drug adjustment and optimization made by expert physicians in treating uncontrolled hypertension (34). Therefore, we feel that our results may be extrapolated to a real-life setting.

\section{Strengths and limitations}

Like in any clinical study, the Hawthorne effect may play a part in the gradual assimilation of the two groups of the Oslo RDN study (35). Receiving close follow-up over an extended time in a tertiary specialist center may contribute to positive behavioral changes, including lifestyle alterations and improved drug adherence. Even though a significant amount of time has passed between randomization and the seven-year follow-up, the Hawthorne effect can affect the results to some degree. Additionally, the Oslo RDN study includes only 19 patients. As a limited number of participants increases the risk of type 2 errors, all statistical output should be interpreted with caution. Between the follow-up visits, patients were cared for by their primary physicians, thus we had little control over any drug adjustments made by other physicians unless the patients contacted our department. As the RDN patients received drug adjustment after one year, the true long-term effect of the RDN procedure itself may have been obscured. Recognizing these weaknesses, we nevertheless have no patients lost to follow-up, and a thorough assessment of all patients on seven-year follow-up, providing a contribution to the long-term effects of patients with difficult-to-treat hypertension.

\section{Conclusions}


We revealed no long-term complications of the RDN procedure, and no functional- or anatomical renal injury seven years after the intervention in either group. As such, RDN seems to be a safe procedure in patients with apparent treatment-resistant hypertension, but it does not seem to yield superior results compared to adjustment of medications after seven years. Intensive follow-up and adjustment of antihypertensive drugs in the first six months resulted in persistent better BP control on long-term follow-up. Drug adjustment should therefore remain the mainstay of treatment.

\section{Disclosure of interests}

The authors disclose no relevant conflicts of interest.

\section{Funding}

This study was funded by The South-Eastern Norway Regional Health Authority.

\section{Acknowledgements}

The authors would like to thank Prof. Emeritus Knut Liestøl at the Department of Informatics, University of Oslo, research bioengineer Vibeke N. Kjær at the Section for Cardiovascular and Renal Research, Oslo University Hospital, Ullevål and Prof. Morten Rostrup at the Department of Behavioral Sciences in Medicine, University of Oslo and Department of Acute Medicine at Oslo University Hospital, Ullevål for their valuable contributions to this work. 


\section{REFERENCES}

1. Krum H, Schlaich M, Whitbourn R, Sobotka PA, Sadowski J, Bartus K, et al. Catheter-based renal sympathetic denervation for resistant hypertension: a multicentre safety and proof-of-principle cohort study. Lancet. 2009;373(9671):1275-81.

2. Kiuchi MG, Esler MD, Fink GD, Osborn JW, Banek CT, Bohm M, et al. Renal Denervation Update From the International Sympathetic Nervous System Summit: JACC State-of-the-Art Review. J Am Coll Cardiol. 2019;73(23):3006-17.

3. Symplicity HTNI, Esler MD, Krum H, Sobotka PA, Schlaich MP, Schmieder RE, et al. Renal sympathetic denervation in patients with treatment-resistant hypertension (The Symplicity HTN-2 Trial): a randomised controlled trial. Lancet. 2010;376(9756):1903-9.

4. Kandzari DE, Bhatt DL, Brar S, Devireddy CM, Esler M, Fahy M, et al. Predictors of blood pressure response in the SYMPLICITY HTN-3 trial. Eur Heart J. 2015;36(4):219-27.

5. Townsend RR, Mahfoud F, Kandzari DE, Kario K, Pocock S, Weber MA, et al. Catheter-based renal denervation in patients with uncontrolled hypertension in the absence of antihypertensive medications (SPYRAL HTN-OFF MED): a randomised, sham-controlled, proof-of-concept trial. Lancet. 2017;390(10108):2160-70.

6. Kandzari DE, Bohm M, Mahfoud F, Townsend RR, Weber MA, Pocock S, et al. Effect of renal denervation on blood pressure in the presence of antihypertensive drugs: 6-month efficacy and safety results from the SPYRAL HTN-ON MED proof-of-concept randomised trial. Lancet. 2018;391(10137):2346-55.

7. Weber MA, Mahfoud F, Schmieder RE, Kandzari DE, Tsioufis KP, Townsend RR, et al. Renal Denervation for Treating Hypertension: Current Scientific and Clinical Evidence. JACC Cardiovasc Interv. 2019;12(12):1095-105.

8. Coppolino G, Pisano A, Rivoli L, Bolignano D. Renal denervation for resistant hypertension. Cochrane Database Syst Rev. 2017;2:CD011499.

9. Mahfoud F, Bohm M, Schmieder R, Narkiewicz K, Ewen S, Ruilope L, et al. Effects of renal denervation on kidney function and long-term outcomes: 3-year follow-up from the Global SYMPLICITY Registry. Eur Heart J. 2019.

10. Fadl Elmula FE, Jin Y, Yang WY, Thijs L, Lu YC, Larstorp AC, et al. Meta-analysis of randomized controlled trials of renal denervation in treatment-resistant hypertension. Blood Press.

2015;24(5):263-74.

11. Kario K, Ogawa H, Okumura K, Okura T, Saito S, Ueno T, et al. SYMPLICITY HTN-Japan - First Randomized Controlled Trial of Catheter-Based Renal Denervation in Asian Patients. Circ J. 2015;79(6):1222-9.

12. Fadl Elmula FE, Hoffmann P, Larstorp AC, Fossum E, Brekke M, Kjeldsen SE, et al. Adjusted drug treatment is superior to renal sympathetic denervation in patients with true treatment-resistant hypertension. Hypertension. 2014;63(5):991-9.

13. Fadl Elmula FE, Hoffmann P, Fossum E, Brekke M, Gjonnaess E, Hjornholm U, et al. Renal sympathetic denervation in patients with treatment-resistant hypertension after witnessed intake of medication before qualifying ambulatory blood pressure. Hypertension. 2013;62(3):526-32.

14. Hjornholm U, Larstorp ACK, Andersen MH, Hoieggen A. Directly observed therapy prior to ambulatory blood pressure measurement (DOT-HTN) in uncontrolled hypertensive patients - Effect on blood pressure, safety and patient perception. Blood Press. 2019;28(5):327-35.

15. Faini A, Omboni S, Tifrea M, Bubenek S, Lazar O, Parati G. Cardiac index assessment: validation of a new non-invasive very low current thoracic bioimpedance device by thermodilution. Blood Press. 2014;23(2):102-8.

16. Mancia G, De Backer G, Dominiczak A, Cifkova R, Fagard R, Germano G, et al. 2007 ESH-ESC Guidelines for the management of arterial hypertension: the task force for the management of arterial hypertension of the European Society of Hypertension (ESH) and of the European Society of Cardiology (ESC). Blood Press. 2007;16(3):135-232. 
17. Mancia G, Fagard R, Narkiewicz K, Redon J, Zanchetti A, Bohm M, et al. 2013 ESH/ESC Practice Guidelines for the Management of Arterial Hypertension. Blood Press. 2014;23(1):3-16.

18. Williams B, Mancia G, Spiering W, Agabiti Rosei E, Azizi M, Burnier M, et al. 2018 Practice Guidelines for the management of arterial hypertension of the European Society of Cardiology and the European Society of Hypertension. Blood Press. 2018;27(6):314-40.

19. Levey AS, Stevens LA, Schmid CH, Zhang YL, Castro AF, 3rd, Feldman HI, et al. A new equation to estimate glomerular filtration rate. Ann Intern Med. 2009;150(9):604-12.

20. Stevens PE, Levin A, Kidney Disease: Improving Global Outcomes Chronic Kidney Disease Guideline Development Work Group M. Evaluation and management of chronic kidney disease: synopsis of the kidney disease: improving global outcomes 2012 clinical practice guideline. Ann Intern Med. 2013;158(11):825-30.

21. Inker LA, Schmid CH, Tighiouart H, Eckfeldt JH, Feldman HI, Greene T, et al. Estimating glomerular filtration rate from serum creatinine and cystatin C. N Engl J Med. 2012;367(1):20-9. 22. Cleophas TJ, Zwinderman AH, van Ouwerkerk BM. Methods for analysing cardiovascular studies with repeated measures. Neth Heart J. 2009;17(11):429-33.

23. Xu W, Goldberg SI, Shubina M, Turchin A. Optimal systolic blood pressure target, time to intensification, and time to follow-up in treatment of hypertension: population based retrospective cohort study. BMJ. 2015;350:h158.

24. Tsujimoto T, Kajio H. Intensive Blood Pressure Treatment for Resistant Hypertension. Hypertension. 2019;73(2):415-23.

25. Lamb EJ, Tomson CR, Roderick PJ, Clinical Sciences Reviews Committee of the Association for Clinical B. Estimating kidney function in adults using formulae. Ann Clin Biochem. 2005;42(Pt 5):32145.

26. Stevens LA, Coresh J, Greene T, Levey AS. Assessing kidney function--measured and estimated glomerular filtration rate. N Engl J Med. 2006;354(23):2473-83.

27. Weinstein JR, Anderson S. The aging kidney: physiological changes. Adv Chronic Kidney Dis. 2010;17(4):302-7.

28. Hommos MS, Glassock RJ, Rule AD. Structural and Functional Changes in Human Kidneys with Healthy Aging. J Am Soc Nephrol. 2017;28(10):2838-44.

29. Glassock RJ, Winearls C. Ageing and the glomerular filtration rate: truths and consequences. Trans Am Clin Climatol Assoc. 2009;120:419-28.

30. Mennuni S, Rubattu S, Pierelli G, Tocci G, Fofi C, Volpe M. Hypertension and kidneys: unraveling complex molecular mechanisms underlying hypertensive renal damage. J Hum Hypertens. 2014;28(2):74-9.

31. Chung M, Leitz FH, Maier G, Patrick JE, Gural RP, Symchowicz S. Rising multiple-dose pharmacokinetics of labetalol in hypertensive patients. J Clin Pharmacol. 1986;26(4):248-52.

32. de Jager RL, van Maarseveen EM, Bots ML, Blankestijn PJ, investigators S. Medication adherence in patients with apparent resistant hypertension: findings from the SYMPATHY trial. $\mathrm{Br} J$ Clin Pharmacol. 2018;84(1):18-24.

33. Gupta P, Patel P, Strauch B, Lai FY, Akbarov A, Gulsin GS, et al. Biochemical Screening for Nonadherence Is Associated With Blood Pressure Reduction and Improvement in Adherence. Hypertension. 2017;70(5):1042-8.

34. Fadl Elmula FE, Rebora P, Talvik A, Salerno S, Miszkowska-Nagorna E, Liu X, et al. A randomized and controlled study of noninvasive hemodynamic monitoring as a guide to drug treatment of uncontrolled hypertensive patients. J Hypertens. 2015;33(12):2534-45.

35. McCambridge J, Witton J, Elbourne DR. Systematic review of the Hawthorne effect: new concepts are needed to study research participation effects. J Clin Epidemiol. 2014;67(3):267-77. 


\section{FIGURE LEGENDS}

\section{Figure 1}

Flowchart illustrating the outline of the Oslo RDN study and the two groups; the Renal Denervation group, and the Drug Adjustment group. The box containing " $1 \mathrm{y}+\mathrm{drug}$ adjustment" denotes in the RDN group the start of drug adjustment in this group. The dotted lines illustrate a planned follow-up visit ten years after randomization.

\section{Figure 2}

Long-term Office Blood Pressure in each of the two groups.

The $\mathrm{x}$-axis plots time, with each study visit marked separately. The y-axis denotes blood pressure in mmHg and is truncated. The blue lines indicate the systolic- (upper line with circular points) and diastolic (lower line with square points) blood pressure of the Renal Denervation group. The red lines with (opposite) triangular symbols indicate the same for the Drug Adjustment group. The asterisk denotes statistical difference between the groups at the corresponding visit.

\section{Figure 3}

Long-term daytime Ambulatory Blood Pressure in each of the two groups.

The $\mathrm{x}$-axis plots time, with each study visit marked separately. The $\mathrm{y}$-axis denotes blood pressure in $\mathrm{mmHg}$ and is truncated. The blue lines indicate the systolic- (upper line with circular points) and diastolic (lower line with square points) blood pressure of the Renal Denervation group. The red lines with (opposite) triangular symbols indicate the same for the Drug Adjustment group. The asterisk denotes statistical difference between the groups at the corresponding follow-up visit. 


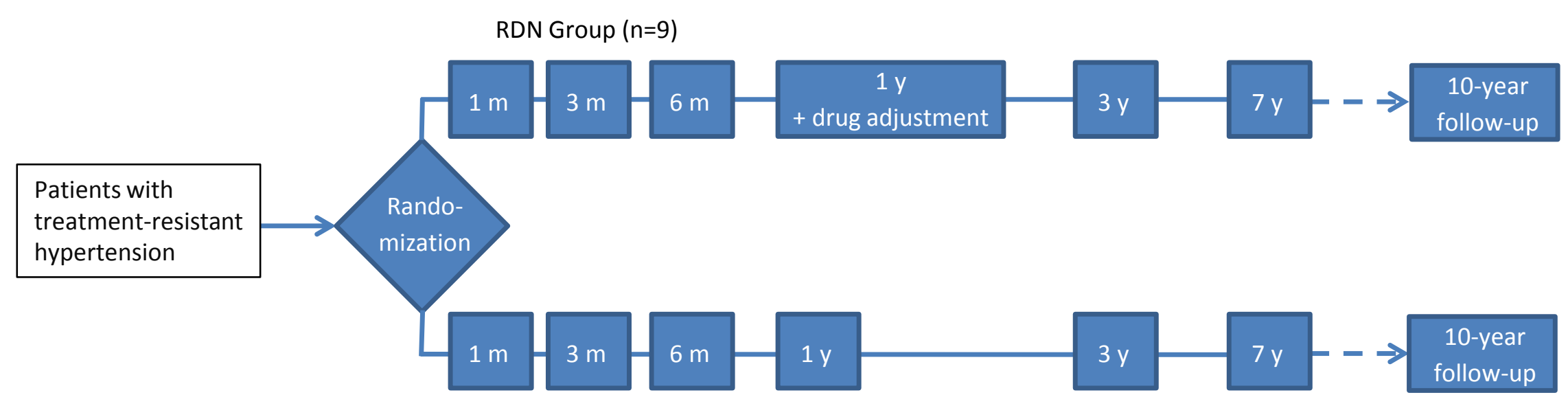

Drug Adjustment Group ( $n=10)$ 
TABLE 1 Group characteristics at randomization

\begin{tabular}{|l|c|c|c|}
\cline { 2 - 4 } \multicolumn{1}{c|}{} & RDN (n=9) & DRUG ADJ. (n=10) & p-value \\
\hline Age in years & $57.0(10.9)$ & $62.7(5.1)$ & 0.16 \\
Gender, \% female & 22 & 0 & 0.21 \\
Body Mass Index, kg/m & $29.0(5.3)$ & $30.0(5.3)$ & 0.72 \\
Coronary Artery Disease (\%) & 11 & 60 & 0.06 \\
Diabetes Mellitus (\%) & 22 & 30 & 1.00 \\
Peripheral Arterial Disease (\%) & 0 & 10 & 1.00 \\
History of Stroke (\%) & 11 & 10 & 1.00 \\
Hypercholesterolemia (\%) & 22 & 40 & 0.63 \\
\hline
\end{tabular}

Data are presented as mean (SD) or percentages.

$R D N=$ Renal Denervation Group, DRUG ADJ = Drug Adjustment Group 


\begin{tabular}{|c|c|c|c|c|c|c|c|c|c|c|c|c|}
\hline & \multicolumn{3}{|c|}{ BASELINE } & \multicolumn{3}{|c|}{6 MONTHS } & \multicolumn{3}{|c|}{3 YEARS } & \multicolumn{3}{|c|}{7 YEARS } \\
\hline & RDN & $\begin{array}{c}\text { DRUG } \\
\text { ADJ. }\end{array}$ & p-value & RDN & $\begin{array}{l}\text { DRUG } \\
\text { ADJ. }\end{array}$ & $p$-value & RDN & $\begin{array}{l}\text { DRUG } \\
\text { ADJ. }\end{array}$ & p-value & RDN & $\begin{array}{l}\text { DRUG } \\
\text { ADJ. }\end{array}$ & p-value \\
\hline Number of antihypertensive agents & $6.0(2.1)$ & $5.0(1.4)$ & 0.50 & $5.8(2.3)$ & $6.0(1.4)$ & 0.45 & $5.1(2.0)$ & $5.4(1.5)$ & 0.60 & $3.9(2.3)$ & $4.7(1.5)$ & 0.49 \\
\hline Angiotensin II receptor blockers (\%) & 100 & 70 & 0.21 & 89 & 70 & 0.58 & 67 & 70 & 1.00 & 56 & 66 & 1.00 \\
\hline Angiotensin-converting enzyme inhibitors (\%) & 33 & 30 & 1.00 & 33 & 30 & 1.00 & 33 & 30 & 1.00 & 33 & 22 & 1.00 \\
\hline Calcium channel blockers (\%) & 89 & 70 & 0.58 & 89 & 80 & 1.00 & 78 & 70 & 1.00 & 77 & 77 & 1.00 \\
\hline$\alpha-1$ blockers (\%) & 56 & 20 & 0.17 & 66 & 50 & 0.65 & 67 & 30 & 0.18 & 44 & 11 & 0.29 \\
\hline$\beta$-blockers (\%) & 56 & 90 & 0.14 & 56 & 70 & 0.65 & 44 & 80 & 0.37 & 22 & 77 & 0.06 \\
\hline Centrally acting sympatholytics (\%) & 56 & 40 & 0.66 & 56 & 40 & 0.66 & 33 & 40 & 1.00 & 11 & 33 & 0.58 \\
\hline Vasodilators (\%) & 0 & 20 & 0.47 & 0 & 30 & 0.47 & 0 & 20 & 0.47 & 0 & 33 & 0.21 \\
\hline Diuretics* (\%) & 100 & 100 & 1.00 & 89 & 100 & 1.00 & 100 & 90 & 1.00 & 88 & 55 & 0.29 \\
\hline Aldosterone antagonists (\%) & 33 & 60 & 0.37 & 22 & 50 & 0.17 & 33 & 50 & 0.65 & 22 & 33 & 1.00 \\
\hline Direct Renin inhibitors (\%) & 22 & 0 & 0.21 & 22 & 0 & 0.21 & 11 & 0 & 0.47 & 0 & 0 & N/A \\
\hline Other potassium-sparing diuretics (\%) & 11 & 0 & 0.47 & 11 & 50 & 0.47 & 11 & 40 & 0.30 & 0 & 33 & 0.21 \\
\hline
\end{tabular}

Data are presented as mean (SD) or percentages of the patients in each group using the specified class of antihypertensive drug.

RDN=Renal Denervation Group, DRUG ADJ= Drug Adjustment Group

*Loop Diuretics and Thiazides 
ABLE 3 Group comparisons of blood pressure, demographic variables, biochemistry and renal function during 7 years of follow-up

\begin{tabular}{|c|c|c|c|c|c|c|c|c|c|c|c|c|}
\hline & \multicolumn{3}{|c|}{ BASELINE } & \multicolumn{3}{|c|}{6 MONTHS } & \multicolumn{3}{|c|}{3 YEARS } & \multicolumn{3}{|c|}{7 YEARS } \\
\hline & RDN & DRUG ADJ. & p-value & RDN & DRUG ADJ. & p-value & RDN & DRUG ADJ. & p-value & RDN & DRUG ADJ. & p-value \\
\hline Body Mass Index (kg/m²) & $29.0(5.3)$ & $30.0(5.3)$ & 0.72 & $29.4(5.4)$ & $30.6(5.1)$ & 0.50 & $29.7(6.4)$ & $30.5(5.6)$ & 0.60 & $30.2(6.7)$ & $31.0(5.8)$ & 0.78 \\
\hline Office Systolic BP (mmHg) & $156(12.6)$ & $160(14.0)$ & 0.55 & $148(7.4)$ & $132(10.2)$ & 0.001 & $147(21.5)$ & $148(32.6)$ & 0.91 & $155(19.3)$ & $146(20.9)$ & 0.39 \\
\hline Office Diastolic BP (mmHg) & 91 (14.9) & $88(12.7)$ & 0.59 & $89(7.9)$ & $77(7.5)$ & 0.004 & $88(11.5)$ & $86(11.8)$ & 0.64 & 93 (12.9) & $85(9.0)$ & 0.14 \\
\hline Ambulatory $24 \mathrm{~h}$ Systolic BP (mmHg) & $149(9.3)$ & $151(11.7)$ & 0.71 & $139(10.5)$ & $130(11.6)$ & 0.11 & $137(13.4)$ & $132(14.8)$ & 0.47 & $140(13.4)$ & $133(13.8)$ & 0.33 \\
\hline Ambulatory $24 \mathrm{~h}$ Diastolic BP (mmHg) & $89(7.2)$ & $85(6.5)$ & 0.19 & $83(3.4)$ & $74(6.7)$ & 0.003 & $81(10.5)$ & $77(6.0)$ & 0.34 & $83(9.6)$ & $78(7.2)$ & 0.22 \\
\hline Ambulatory Daytime Systolic BP (mmHg) & $152(10.4)$ & $152(11.7)$ & 0.98 & $142(10.3)$ & $133(11.3)$ & 0.08 & $139(16.1)$ & $134(14.9)$ & 0.46 & $145(14.8)$ & $137(13.4)$ & 0.27 \\
\hline Ambulatory Daytime Diastolic BP (mmHg) & $93(7.7)$ & $88(8.2)$ & 0.17 & $86(4.4)$ & $77(8.0)$ & 0.008 & $83(12.1)$ & $80(6.6)$ & 0.42 & $87(10.3)$ & $82(8.1)$ & 0.28 \\
\hline Ambulatory Nighttime Systolic BP (mmHg) & $141(9.4)$ & $146(15.9)$ & 0.44 & $132(11.5)$ & $123(13.0)$ & 0.11 & $130(11.4)$ & $128(15.1)$ & 0.69 & $127(12.0)$ & $125(15.4)$ & 0.85 \\
\hline Ambulatory Nighttime Diastolic BP (mmHg) & $82(7.2)$ & $79(4.5)$ & 0.29 & $76(4.8)$ & $67(5.8)$ & 0.002 & $75(9.3)$ & $72(6.4)$ & 0.34 & $74(9.8)$ & $70(9.0)$ & 0.43 \\
\hline Hemoglobin $(g / d L)$ & $15.4(1.3)$ & $15.1(1.3)$ & 0.66 & $14.2(1.6)$ & $13.8(1.4)$ & 0.57 & $14.9(1.4)$ & $15.0(1.3)$ & 0.86 & $15.0(1.2)$ & $14.9(1.4)$ & 0.90 \\
\hline Serum Total Cholesterol (mmol/L) & $5.1(0.8)$ & $4.4(0.8)$ & 0.09 & $4.5(0.8)$ & $5.2(3.5)$ & 0.84 & $4.5(0.8)$ & $4.4(1.4)$ & 0.74 & $3.9(0.7)$ & $3.6(0.6)$ & 0.33 \\
\hline Serum HDL (mmol/L) & $1.2(0.3)$ & $1.4(0.2)$ & 0.40 & $1.1(0.3)$ & $1.2(0.3)$ & 0.73 & $1.2(0.2)$ & $1.3(0.4)$ & 0.66 & $1.2(0.2)$ & $1.2(0.2)$ & 0.75 \\
\hline Serum LDL (mmol/L) & $3.6(0.8)$ & $2.7(0.8)$ & 0.02 & $2.9(0.8)$ & $2.6(1.2)$ & 0.48 & $3.0(0.8)$ & $2.7(1.2)$ & 0.56 & $2.4(0.7)$ & $2.1(0.5)$ & 0.25 \\
\hline Serum Triglycerides (mmol/L) & $1.3(0.5)$ & $1.2(0.5)$ & 0.79 & $1.2(0.6)$ & $1.6(0.6)$ & 0.14 & $1.5(0.6)$ & $1.5(1.0)$ & 0.60 & $1.4(0.3)$ & $1.4(0.6)$ & 0.96 \\
\hline Serum Potassium (mmol/L) & $3.7(0.4)$ & $3.9(0.3)$ & 0.18 & $3.7(0.3)$ & $3.8(0.3)$ & 0.34 & $4.0(0.5)$ & $4.0(0.4)$ & 0.95 & $4.0(0.5)$ & $3.9(0.3)$ & 0.89 \\
\hline Serum CRP (mg/L) & $2.4(1.7)$ & $3.3(2.5)$ & 0.41 & $3.1(4.6)$ & $2.5(3.1)$ & 0.60 & $2.5(2.1)$ & $4.8(5.0)$ & 0.22 & $2.2(1.2)$ & $7.1(6.9)$ & 0.05 \\
\hline Serum Creatinine $(\mu \mathrm{mol} / \mathrm{L})$ & $91.6(6.7)^{*}$ & $88.9(12.2)$ & 0.68 & $92.0(12.3)^{*}$ & $101.0(22.0)$ & 0.25 & $89.8(12.6)^{*}$ & $93.7(20.8)$ & 0.60 & $87.4(11.7)^{*}$ & $105.8(36.5)$ & 0.26 \\
\hline Calculated eGFR by CKD-EPI (mL/min/1.73m²) & $90.1(10.0)^{*}$ & $89.1(6.6)$ & 0.80 & $90.4(11.5)^{*}$ & $85.0(9.4)$ & 0.28 & $91.3(10.7)^{*}$ & $87.8(8.4)$ & 0.45 & $76.3(14.2)^{*}$ & $66.9(22.3)$ & 0.33 \\
\hline Urine Albumin/Creatinine Ratio $(\mathrm{mg} / \mathrm{mmol})$ & $2.4(3.0)$ & $2.0(2.4)$ & 0.44 & $1.5(2.1)$ & $1.2(1.3)$ & 0.78 & $1.5(2.1)$ & $4.1(5.0)$ & 0.70 & $3.3(4.4)$ & $5.8(11.1)$ & 0.55 \\
\hline
\end{tabular}

Data presented as mean (SD). Significant p-values in bold print.

*1 Outlier omitted.

$R D N=$ Renal Denervation Group, DRUG ADJ= Drug Adjustment Group, BP=Blood Pressure, $H D L=H i g h$-Density Lipoprotein, $L D L=L o w$-Density

Lipoprotein, CRP=C-Reactive Protein eGFR=Estimated Glomerular Filtration Rate, CKD-EPI = Chronic Kidney Disease Epidemiology Collaboration 


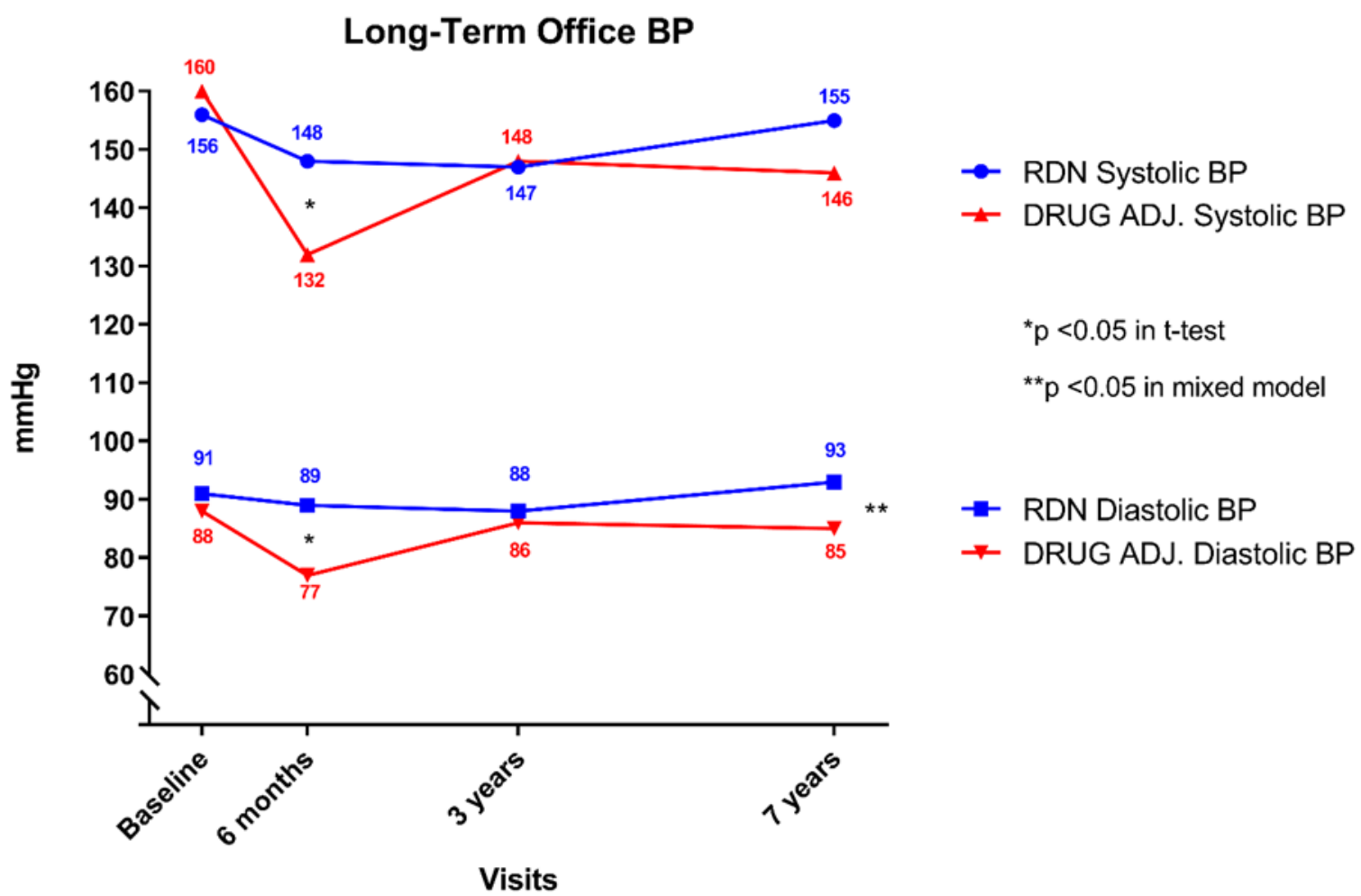

Long-Term Daytime Ambulatory BP

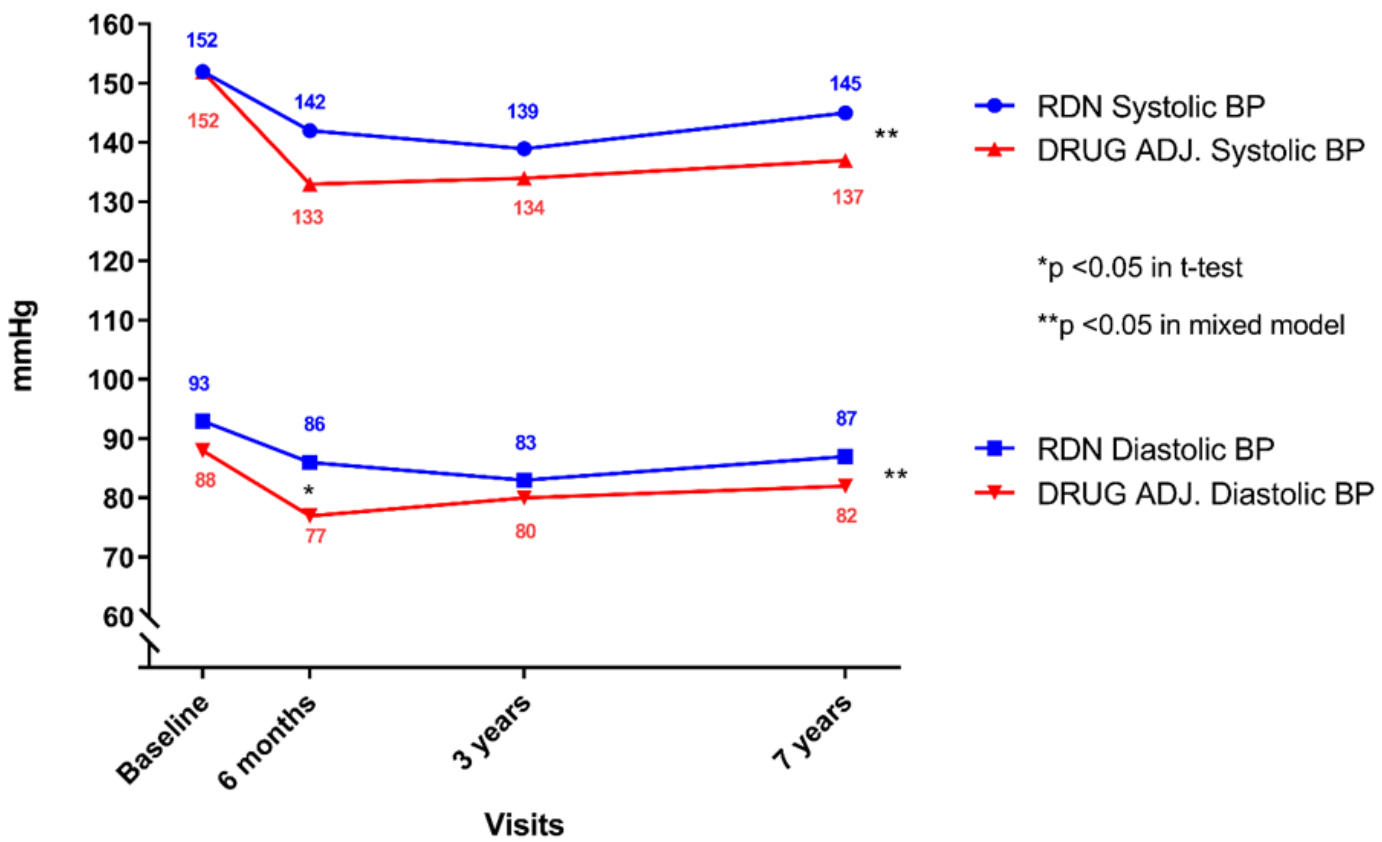

